Investigaciones Fenomenológicas, vol. Monográfico 5, 2015, 287-299. e-ISSN: $1885-1088$

\title{
APROXIMACIÓN HERMENÉUTICO-CRÍTICA \\ A LA FENOMENOLOGÍA DE LA RAZÓN EN IDEAS I
}

\author{
Hermeneutical-Critical Approach \\ to the Phenomenology of Reason in IDEAS I
}

\author{
Balbino Quesada \\ IES San Juan de La Cruz de Úbeda \\ qtalavera67@yahoo.es
}

\begin{abstract}
Resumen: La fenomenología de la razón escudriña todos los procesos racionales que intervienen en la aprehensión del objeto real y de su constitución, y las consiguientes relaciones noético-noemáticas. Sólo la razón pura colegida, primero como fuerza que depura los procesos de la evidencia y las posiciones del sujeto, y sólo la razón entendida también como proceso que ratifica la verificabilidad del darse el objeto y de todos los demás procesos y relaciones, se revela como la única instancia posible capaz de asegurar la validez del conocimiento y del objeto del conocimiento. Por otro lado, la razón juega un papel decisivo en lo que respecta al sujeto de razón. Ésta es la nota constitutiva y respectiva del ser humano, el poder que lo separa del entramado de facticidades y contingencias de las que se compone la realidad, de suerte que éste no es un factum más entre todas las objetividades, tal como postulan la psicología y las ciencias naturales. El hombre es un ser mundano, está efectivamente en el mundo. Ahora bien, "su ser en" y "su estar en" son modificados por el poder de la razón. Su modo de ser mundano es serlo racionalmente. Únicamente de este modo el Umwelt deviene Lebenswelt.
\end{abstract}

\begin{abstract}
The phenomenology of reason inspects all rational processes involved (which participate) in the apprehension of real object and its constitution, and the respective noeticnoematic relationships. Only the pure reason comprehended, first of all, as a strength which purifies the mentioned processes, the evidence and the subject positions. Secondly the reason, also comprehended as a process which confirms the object verificability which gives itself and the other processes and relationships, appears as the only possible power, which is able to guarantee the validity of the knowledge, and so the object of knowledge. On the other hand, the reason plays a very important role in the subject of the reason. This is the constitutive and respective note of the human being, the power (strength) that separates it from the labyrinth of facts and contingencies which makes up reality, so that this (human being) isn't a fact among all objectivities, as postulated by psychology and natural sciences. The human being is a worldly being; he is really in the world. Now then, "his being-in" and "his staying-in" are modified by the strength of the reason. His way of being worldly is being rationaly. Only in this way the "Umwelt" becomes "Lebenswelt".
\end{abstract}




\section{PreÁmbulo}

Permítanme compartir con todos ustedes algunas ideas, siempre provisionales, acerca de las cuestiones que Husserl plantea en la fenomenología de la razón de Ideas I. Nos referimos a este tema con la expresión «problema de la racionalidad», precisamente porque estamos persuadidos de que la pretensión del propio filósofo no era tanto agotar una fenomenología de la razón, cuanto presentar una teoría de la racionalidad fenomenológica al hilo de las reflexiones que son llevadas a cabo en las páginas que preceden a la sección cuarta, cuyo segundo capítulo es «Fenomenología de la razón».

Presentar una teoría de la racionalidad en términos de problema no implica aludir a una naturaleza incierta o a una forma indeterminada de la razón, sino que, por el contrario, apunta a la necesidad de una reflexión permanente de la esencia propia de la filosofía, si (y en la medida en) es que efectivamente la razón conforma la posibilidad esencial de la filosofía.

Entendemos que de la lectura de Ideas I se desprende que la filosofía sigue manteniendo con todo vigor y con toda la fuerza de sus orígenes su función primigenia de dar razón con firmeza de ciencia estricta precisamente de todo aquello que en torno al ser humano y a su sí-mismo acontece. Mas esta idea de la filosofía constituye, al menos para quien escribe estas líneas, el sentido genuino de la vida filosófica entendida como verdad; constituye, asimismo, el sentido de la vida filosófica vivida desde el libre ejercicio del ser pensante, del ser humano en tanto que sujeto entitativo de razón. En este sentido, Zubiri afirmaba que «el que se dedica a la realidad verdadera [...] no posee verdades, sino que está poseído por ellas» ${ }^{1}$.

\section{LA RAZÓN Y EL CAMINO DEL SABER}

De los tres capítulos que componen la sección cuarta de Ideas I, el primero, «El sentido noemático y la referencia al objeto», trata de arrojar luz al tradicionalmente oscuro ámbito de las relaciones del objeto (lo realmente existente "en

\footnotetext{
${ }^{1}$ La cita pertenece al discurso de aceptación del premio "Santiago Ramón y Cajal" (19 de octubre de 1982), con el que Zubiri fue galardonado. Está recogido en Escritos menores (1953-1983), ed. y publicación de Germán Marquínez Argote, Alianza Editorial - Fundación Zubiri, Madrid, 2007, 322.
} 


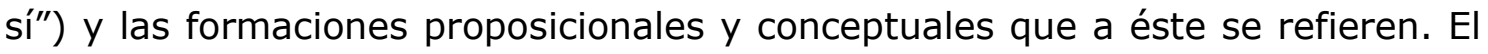
segundo, «Fenomenología de la razón», desarrolla una teoría de la "racionalidad ideal", una teoría de la razón, en la que el sujeto de la conciencia racional comprueba y fundamenta lo mentado del objeto, el acto judicativo acerca del objeto, y "ve directamente" (o intuye) el ser real en tanto que real. Es decir, se teje una fenomenología de la razón que se sanciona inequívocamente la correlación entre lo mentado del ser y el ser real, de suerte que la razón fenomenológica es intuida como "posibilidad de comprobación inequívoca" del ser real en tanto que real. El tercero, «Grados de universalidad en los problemas de la teoría de la razón», pone de manifiesto la singular riqueza de la razón (ideal) en lo que concierne a las ampliaciones de la descripción de la relación ser realser mentado y a las extensiones amplificativas con la ontología formal y la ontología regional.

Podríamos caracterizar el concepto de razón en el contexto de Ideas I como un patrón necesario por el que todo el saber acerca del objeto ha de ser medido para poder ser verdadero ${ }^{2}$. Si nos atenemos a esta incipiente definición, observamos que la razón guarda una estrecha relación con el conocimiento, el saber, la realidad y la verdad, y aún antes con el sujeto del conocimiento.

La razón se colige como "el" ingrediente necesario y privativo del ser humano que garantiza la indubitable certeza del saber propio y la veracidad de aquello que conocemos. Pero la razón hay que situarla en el horizonte de la filosofía como ciencia universal, y, por ende, como philosophia perennis, que sobrepasa definitivamente la estrechez de miras de las meras ciencias de hechos. Es más, la dilucidación de la cuestión de la razón fenomenológica implica la emancipación del filósofo $-y$, por tanto, de todo ser humano- de la hegemonía de la facticidad y, consecuentemente, la justificación de su tarea como valedor de una filosofía nueva orientada al esclarecimiento total del ser humano racional no sujeto a contingencias, «como el verdadero lugar originario del sentido del mundo» ${ }^{3}$, de los fenómenos en sí mismos considerados, y de su cono-

\footnotetext{
2 «En el saber -afirma Husserl- poseemos la verdad»; Logische Untersuchungen. Erster Theil. Prolegomena zur reinen Logik, [En línea]. Halle a. S., M. Niemeyer, 1900, http://www.freidok.unifreiburg.de/volltexte/5920, 12; Logische Untersuchungen. Erster Band, [En línea] 1913², http://www.freidok.uni-freiburg.de/volltexte/609612; Investigaciones lógicas I; ed. de García Morente y J. Gaos; Alianza Editorial, Madrid, 2009, 41.

${ }^{3}$ San Martín, J., La fenomenología como teoría de una racionalidad fuerte. Estructura y función de la fenomenología de Husserl y otros ensayos, UNED, Madrid, 1994, 162.
} 
cimiento cierto ${ }^{4}$. En definitiva, justifica la existencia del filósofo como valedor de la verdad. Con tinte dramático expresa su quehacer el propio Husserl el 25 de septiembre de 1906:

Mencionaré en primer lugar la tarea general que tengo que resolver para mí mismo si pretendo llamarme filósofo. Me refiero a una crítica de la razón [...]. Sin dilucidar, en rasgos generales, el sentido, la esencia, los métodos, los puntos de vista capitales de una crítica de la razón; sin haber pensado, esbozado, averiguado y demostrado un bosquejo general de ella yo en verdad no puedo vivir sinceramente. Bastante he probado los suplicios de la oscuridad, de la duda que vacila de acá para allá. Tengo que llegar a íntima firmeza. Sé que se trata de algo grande, inmenso ${ }^{5}$.

Husserl toma como punto de partida del itinerario de una fenomenología de la razón la realidad, el objeto. La pregunta es inevitable: ¿por qué comenzar por la realidad, si lo que se pretende es una crítica de la razón? ¿Acaso no sería más preciso comenzar con un análisis descriptivo de ésta, independientemente de la realidad? ¿No convendría mejor considerar a la razón y al ser humano, en tanto que sujeto racional, sin atender en primera instancia aquel ámbito que se halla extra muros del propio ser humano? La respuesta que el filósofo da a estas preguntas no parece dejar duda:

todo lo que llamamos objeto [Gegenstand], aquello de que hablamos, lo que como realidad tenemos ante los ojos, lo que tenemos por posible y probable, lo que nos figuramos por imprecisamente que sea, es, sólo con el ser tal, un objeto de conciencia, y esto quiere decir que, sean y se llamen mundo y realidad lo que sean y se llamen, tiene que estar representado dentro del marco de la conciencia real y posible por sentidos, o proposiciones, llenos por el correspondiente contenido más o menos intuitivo ${ }^{6}$.

Hay que remontarse a las secciones segunda y tercera respectivamente para comprender en buena lid la razón por la que Husserl llega a la conclusión que acabamos de señalar, a saber, el objeto real -es decir, existente en el

\footnotetext{
${ }^{4}$ Ibíd., 148.

${ }^{5}$ Die Idee der Phänomenologie, Husserliana II, Herausgegeben und Eingeleitet von W. Biemel, den Haag, Martinus Nijhoff, 1950, VII; (La idea de la fenomenología ((trad. de Miguel García-Baró), FCE, México, 2004, 14).

${ }^{6}$ Ideen zu einer reinen Phänomenologie und phänomenologischen Philosophie. Erstes Buch: Allgemeine Einführung in die reine Phänomenologie [En línea]. Halle, M. Niemeyer, 1913, http://www.freidok.unifreiburg.de/volltexte/5973, 278. (Trad. cast., Ideas relativas a una fenomenología pura y a una filosofía fenomenológica. Libro I, FCE, México, 19932, 321). En adelante, la primera notación del año de publicación y la página se referirán a la edición alemana; la segunda, a la española. Cursiva mía.
} 
tiempo- es término de la conciencia, y con ello parece más claro poder entender la necesaria recurrencia a la realidad para referirse a la razón. Lo indicaremos con la mayor brevedad posible: cuando la realidad, el mundo, cae bajo el reino de la desconexión [Ausschaltung], «toda realidad es re(con)ducida, o llevada a la vida subjetiva sólo en la cual la realidad tiene sentido» ${ }^{7}$. Sin embargo, para que la realidad pueda ser re(con)ducida a la vida subjetiva, tal realidad qua real, necesariamente independiente de toda conciencia para poder mantener el estatuto ontológico de real, se ha dado de manera absoluta en la experiencia a la conciencia. Sólo manteniendo la dación o autodonación de la realidad en la experiencia, tal realidad no es una mera representación, sino la realidad en sí misma considerada, cuyo sentido se halla en la vida subjetiva. De este modo, tampoco el mundo es el de la representación, sino un mundo cuyo sentido es netamente humano, porque ha sido reducido a la conciencia absoluta mediante la reducción trascendental. Así pues, podemos dirigir la mirada a la razón referida a la realidad, y, en consecuencia, podemos realizar una crítica de la razón, teniendo en cuenta fundamentalmente que «la razón es ante todo donación de algo en su propia carne, es decir, en sí mismo» ${ }^{8}$. En virtud de este aserto, estamos en disposición de abordar la cuestión de "la razón como ingrediente netamente humano", tal como lo denominamos supra, pero en el modo en que el ser humano es sujeto de la razón. En efecto, podríase entender de las palabras citadas que la razón es "lo donado" en tanto que donado, es decir, no el contenido de la donación, sino "lo donado mismo". Pues bien, si consideramos "lo donado" en sí mismo o si lo valoramos en tanto que contenido, caemos en el error de atribuirle a la realidad la razón, de suerte que ésta pertenecería a la realidad: ésta se da en la pura experiencia. Pero no sería correcta tal interpretación, porque la razón es primordialmente el acto mismo de aprehensión por parte de la conciencia trascendental (del yo trascendental), de la realidad absoluta, «reduciéndome a la realidad tal como se da y se puede dar en la experiencia» ${ }^{9}$. Efectivamente, la donación es posible porque el yo trascenden-

\footnotetext{
7 San Martín, J., La fenomenología como teoría..., op. cit,. 38.

8 Ibíd., 39; vid., nota 11.

${ }^{9}$ Ibíd., 38. La profesora López Sáenz, Ma. C. establece una serie de consideraciones en torno a la experiencia en Husserl, que nos parecen sumamente interesantes, entre otras cosas, porque abren una nueva perspectiva de la propia Erfahrung husserliana. Anclada en la vida, la experiencia, efectivamente, es una actividad y, en tanto que tal, dinámica; y como consecuencia de esta consideración, no es forzado caracterizarla como «actividad creadora»; vid., Corrientes actuales de la filosofía I. En-clave fenomenológica, UNED-Dykinson, Madrid, 2012, 83.
} 
tal, aquél que ha superado la mera representación de lo real y se ha colocado en un plano trascendente, se reduce a la realidad, y se halla, pues, en disposición de incorporar la realidad como dada a la propia vida. Aprehensión y donación son los dos componentes, las dos capas, que conforman el núcleo o esencia de la conciencia trascendental o de la vida subjetiva. A esto que acabamos de señalar, el profesor San Martín lo denomina «estructura absoluta», y está compuesta por «vida subjetiva-realidad mundana»; es absoluta en la medida en que constituye «el principio sólo a partir del cual se puede iniciar cualquier análisis y planteamiento filosófico [...]; es una estructura inderivable, porque todo se deriva de ella» ${ }^{10}$. Aprehensión y donación se insertan indefectiblemente en la razón, y por ello hablamos de la razón como ingrediente netamente humano: la razón se cifra positivamente en la vida subjetiva, que da sentido al mundo, en la conciencia trascendental que aprehende el mundo en tanto que mundo y le otorga un sentido humano.

Consideremos ahora brevemente los conceptos "contenido" [Inhalt] y "sentido" [Sinn]. Distinguimos, de una parte, el «contenido de la conciencia» [Bewußtseinsinhalt] y, de otra, el «núcleo noemático» [noematischen Kerns]. Entendemos por «contenido de conciencia», según lo expresa Husserl, «conciencia de algo [Bewußtsein von etwas], vivencia intencional [intentionale Erlebnis], referencia a un objeto [Beziehung auf Gegenständliches]» ${ }^{11}$. Es decir, el contenido de conciencia es el "algo" que pertenece a la conciencia, en tanto que ésta se refiere a ese "algo" como "su" algo. Mas el "algo" se constituye en la conciencia como "sentido" y no como "algo", de suerte que el contenido de la conciencia es el sentido. Por ejemplo, veo un árbol en el jardín, tengo conciencia del árbol. La conciencia aprehende, por así decirlo, el sentido, no el objeto; tal es su modo de referirse a un objeto. Por otro lado, entendemos que el «núcleo noemático» o contenido noemático es «un íntimo elemento [innerstes Moment] del nóema» ${ }^{12}$. El nóema no es paralelo a la conciencia, de manera que la referencia del nóema es la nóesis y no el objeto; en otras palabras, la conciencia contiene elementos téticos como sentido del "algo", mientras que el nóema "contiene" el «"lo que" intencional y pleno» ${ }^{13}$ que es la nóesis respecto del

\footnotetext{
${ }^{10}$ La fenomenología como teoría..., op. cit., 39.

${ }^{11}$ Ideen, op. cit., 1913, 268; 19932, 309.

${ }^{12}$ Ibíd., 1913, 268-269; 19932, 310.

13 Ibíd., 1913, 268; 19932, 309.
} 
nóema. El nóema se diferencia de la conciencia por el contenido: ésta contiene caracteres téticos y aquél contiene «el núcleo temático» que ha sido caracterizado por los caracteres téticos. El núcleo temático es el «innerstes Moment» del nóema, es decir, «funciona como "soporte" de propiedades noemáticas que le son especialmente inherentes, a saber, de las propiedades, noemáticamente modificadas, de "lo mentado en cuanto tal"»14.

Se deduce de lo que antecede (respecto del contenido) que lo que para la conciencia es «sentido» no lo es para el nóema. El sentido noemático, según Husserl, es «una esencia insensible a tales modificaciones» ${ }^{15}$, que la conciencia establece en su referencia al objeto intencional. El contenido noemático es el correlato del objeto dado en la experiencia absoluta. De ahí que Husserl se refiera con todo rigor y precisión al «contenido noemático» como «sentido» noemático y a éste como la «esencia», en tanto que es invariable respecto de toda modificación.

\section{LA POSIBILIDAD DE UNA ONTOLOGÍA EN EL PROYECTO DE LA FENOMENOLOGÍA DE LA} RAZÓN. LA INTUICIÓN

Si precisamos más qué es "el objeto dado en la experiencia absoluta" una vez que se ha practicado la reducción, podemos poner rumbo en una «dirección hacia el objeto percibido» ${ }^{16}$, que requiere un paso previo, a saber, la forma en que un objeto se da a la conciencia. En el darse a ésta el objeto deviene "objeto-de-la-conciencia" y como tal es constituido. Esto es lo que en fenomenología se denomina "conciencia intencional": la conciencia se dirige al objeto. El "ser objeto de" (la conciencia) equivale al modo de darse el objeto, es decir, es su contenido. Y la forma de ser "contenido de" (la conciencia) es el sentido"17. Por tanto, el contenido de la conciencia y el contenido noemático son paralelos, por lo cual el sentido, esto es, "lo mentado" -el objeto-, responde exactamente al mismo e idéntico objeto.

\footnotetext{
${ }^{14}$ Ibíd., 1913, 269; 19932, 310.

15 Ibíd., $1993^{2}, 310$.

${ }^{16}$ Es esto justamente la «intuición categorial», según López Sáenz, Ma. C.; Corrientes.., op. cit., 82.

17 Por ello, el sentido no es una esencia, sino una «forma abstracta»-afirma Husserl- «a un segundo concepto del "objeto en el cómo" -en el cómo de sus modos de darse». Y lo mismo vale para el nóema: «el sentido no es [...], sino una especie de forma abstracta inmanente a él [el nóema]»; Ideas I, op. cit., 1913,$273 ; 1993^{2}, 315$
} 
Estamos persuadidos de que la cuestión del ser tiene en la fenomenología una importancia capital, porque lo que se pretende poner de manifiesto es la realidad, el objeto real, el verdadera y objetivamente existente. Efectivamente, la fenomenología no habla del ser real del objeto, sino del ser real el objeto. Tal es su esencia: su ser-real. ¿Acaso ser y realidad no son equivalentes? No, si entendemos el ser como un atributo referido a un objeto. En tal caso, ser se circunscribiría más bien a la esfera lingüístico-proposicional y no a un carácter propio del objeto. Ahora bien, si entendemos el ser como como la "nota" por la cual el objeto es $y$, en tanto que es, es real y, por tanto, su realidad se concreta en su existencia efectiva, entonces el ser es equivalente a realidad. De esta manera de entender el ser colegimos no el ser del objeto, sino su "es" actual y actuante. Comprendemos, asimismo, que el ser no es tampoco un trascendental del objeto, sino que es el objeto mismo "siendo". Pues bien, el "siendo" del objeto, su estar en un tiempo y en un espacio determinados, es la nota de inherencia del objeto, por la cual éste es real en tanto que real. Pero el carácter de real no es la materia del objeto, su sustancia, ni tampoco su predicado. Husserl, en efecto, considera que un análisis fenomenológico del ser desde el punto de vista únicamente noético es necesario pero unilateral, por eso propone, mutatis mutandis, la consideración noemática, es decir, la del «núcleo de sentido», su «esencia intencional» ${ }^{18}$.

¿Qué es el «ser verdaderamente»? Éste se ejemplifica a modo de definición mediante el «objeto verdaderamente existente» («wahrhaft seiender Gegensteand $\gg)^{19}$. Ahora bien, parece que la dificultad que encuentra la fenomenología para una analítica del ser estriba en la imposibilidad de darse el ser adecuadamente en un aparecer cerrado. Si el ser es efectivamente un "objeto trascendente" en el sentido de que no se puede dar en sí mismo en una conciencia cerrada, sino que precisa de determinaciones ulteriores, nos preguntamos por su puro sentido noemático, por las leyes esenciales que rigen su contenido y sus relaciones noético-noemáticas. Pues bien, -si como afirma Husserl- «todo está diseñado a priori» ${ }^{20}$ y «todo está precisa y esencialmente motivado» ${ }^{21}$, el continuo de darse el objeto trascendente no es ajeno ni al a priori ni a la esencial

\footnotetext{
${ }^{18}$ Cfr., Ibíd., 1913, 267; 19932, 309.

19 Ibíd., 1913, 296; $1993^{2}, 340$.

${ }^{20}$ Ibíd., 1913, 297; 19932, 341.

${ }^{21}$ Ibíd., 1913, 288; 19932', 331.
} 
motivación racional, de manera que al menos es pensable la idea del continuo y también la idea del darse completamente, que es inherente al continuo. De ahí que la conclusión no sea otra que en la conciencia trascendental se constituye la idea de un ser tal. Del mismo modo, esta misma idea no excluye su evidencia intelectual: al menos ha de poder darse originariamente una idea de que tal modo de ser objeto es concebible 22 .

¿Se desprende de lo anterior que el filósofo propone una idealización de aquellos objetos que no son perceptibles adecuadamente, y que no son evidentes en sí mismos? Afirmamos que no. Husserl no propone la idealización como una salida al problema de la "oscuridad perceptiva" por la falta de una intuición originaria, sino que lo que propone, al hilo de la fenomenología descriptiva, es

que lo real, en la medida en que es la totalidad de la experiencia efectiva y posible, siempre es más que la experiencia que de ella tengo [...], siempre está más allá de mi experiencia efectiva; en definitiva, que es un camino de experiencias posibles; así, la trascendencia, sin ser destruida, queda asumida, es decir, reconducida a la experiencia del sujeto ${ }^{23}$.

Recuperamos ahora el concepto de razón referido al ser, ya que la elucidación racional referida al ser o tiene que ver con el ser verdaderamente o, de lo contrario, el contenido noemático no está relacionado con la nóesis. Y precisamente Husserl afirma que todo objeto verdaderamente existente tiene como correlato el ser que hay que poner racional y originariamente; y además estas correlaciones se dan en una evidencia intelectual. Por otro lado, a todo objeto verdaderamente existente corresponde una conciencia referida a tal objeto, una conciencia en la que el objeto es aprehensible originaria y adecuadamente ${ }^{24}$.

Mas lo que, en definitiva, se ventila en la ontología fenomenológica es una fenomenología de la razón, o lo que es igual, una crítica de la razón ligada al imperio de los meros hechos. En este dominio de la facticidad el objeto campa por los mismos fueros que el sujeto. Pero en una ontología fenomenológica el objeto está reducido al nivel en el que es un objeto del sujeto, es decir, al nivel en el que tiene un sentido para el sujeto ${ }^{25}$. Así, el objeto está significativamente

\footnotetext{
${ }^{22}$ Cfr., Ibíd., 1913, 298; 1993², 342.

${ }^{23}$ San Martín, J., La fenomenología de Husserl como utopía de la razón. introducción a la fenomenología, Biblioteca Nueva, Madrid, 2008, 73.

${ }^{24}$ Cfr., Ideas I, op. cit., 1913, 296; 19932, 340.

25 Cfr., San Martín, J., La fenomenología de Husserl como utopía..., op. cit., 86.
} 
relacionado con las ideas de verdad, razón y conciencia ${ }^{26}$, y si es posible la dilucidación fenomenológica del objeto es sólo desde la legitimidad racional de la posición del sujeto de conciencia respecto del ser verdaderamente, porque, ante todo, el objeto no es sino «un nombre para las relaciones esenciales de la conciencia» 27 .

Estas últimas consideraciones nos conducen de nuevo a la cuestión del sentido (su completitud) y a la de la posición o tesis racional. Una tesis es una vivencia, un acto de apercepción de lo que está "ahí", "delante". En cualquier caso, podemos establecer la posición como guía hacia la conciencia racional y la evidencia, que es el modo de la forma de la conciencia racional, para un análisis de la razón. No en balde, la posición se halla orientada de suyo a poder calificar los actos como tales actos racionales. Ahora bien, si atendemos a la invitación de Husserl y fijamos la atención en los análisis sobre la «completitud de sentido» -sobre el cómo esté lleno o no lleno-, convendría que nos detuviésemos en una porposición extraordinariamente interesante: «un modo de vida del sentido es el intuitivo» ¿Por qué la intuición es un modo de vida del sentido [noemático]? Vivir intuitivamente el sentido es la consecuencia necesaria de haber practicado la epojé: vivimos la realidad en los términos en que justamente nos es dada. De ahí que Husserl se refiera a este modo como «es consciente en el modo del "en persona"» («ist in der Weise des >leibhaft< bewußt») ${ }^{28}$; modo que reviste un carácter preciso: el carácter del leibhaft, y «el sentido con este carácter funciona como base del carácter de posición noemático, o lo que aquí quiere decir lo mismo, el carácter de ser» ${ }^{29}$. El «carácter del en-persona» y correlativamente el «carácter der ser» confieren a la posición, a los actos téticos, un "de suyo" (o nota) distintivo y especial: el de ser racional.

De la mano de estos análisis, nos adentramos en la vía intuitiva. Mas lo que queremos brevemente señalar es que un análisis de la intuición nos lleva a la fenomenología de la razón. Éste es un camino muy interesante ${ }^{30}$ que permitiría abrir otras vías de análisis acerca de la fenomenología de la razón.

Vamos a tratar de sintetizar unas cuantas ideas al respecto de esto último. Justamente, detrás de toda tesis racional está la evidencia originaria, que es un

\footnotetext{
${ }^{26}$ Cfr. Ibid.

${ }^{27}$ Ideas I, op. cit., 1913, 302; 19932 347.

28 Ibíd., 1913, 283; 1993², 326.

${ }^{29}$ Ibíd.

30 Vid., Corrientes..., op. cit, 84-87.
} 
ver apodíctico, un "ver de esencias". La cuestión de la esencia comprende, tal y como está recogido en el primer capítulo de Ideas I, otra cuestión: la de las ontologías regionales. Éstas son formas universales delimitadas por la universalidad material esencial, y su campo de investigación se halla determinado por el propio límite de estas formas.

Pero "las cosas" están insertas en un sistema implicativo de relaciones esenciales, que se plasman en la apofántica o síntesis predicativas puras, que es necesario describir, analizar y conocer tanto en su estructura noética como noemática, amén de las consiguientes relaciones noético-noemáticas. Tales relaciones se subordinan a unas leyes esenciales lógico-formales. Pero, por otro lado, existen leyes ontológico-formales, equivalentes a aquéllas, cuyo ámbito es el propio de las relaciones de las «formas ónticas» ${ }^{31}$ que se dan a la conciencia. Digamos, pues, que con la expresión "formas ónticas" el autor se refiere al serreal del objeto, a su forma de ser, por tanto, a la esencia. La particularidad propia de estas relaciones nos sitúan en un nuevo aspecto de la verdad y de la razón: lo que se persigue ahora es la certeza racional y la verdad de un campo universal formal, derivado de un sistema lógico-predicativo y de una verdad lógica.

La idea de "cosa" está representada en la conciencia por el concepto "cosa". Compuesta de cierto contenido noemático ${ }^{32}$. Comoquiera que la «representación verbal» de la cosa tal como justamente la tenemos se halla en el inicio del proceso, generamos ${ }^{33}$ «representaciones intuitivas»-asevera Husserl- de esta misma cosa, ya que el fin es su «representación universal». Las representaciones intuitivas son naturalmente universales. El modo de proceder de tales representaciones es mediante ejemplos, en cuya virtud construimos «intuiciones imaginativas» de las cosas y representaciones de ellas. Éstas, arguye Husserl, son tan perfectamente reales como aquellas otras representaciones de la experiencia. El autor propone el método de «ideación», en virtud del cual se aprehende la «esencia "cosa"»y se aprehende con «intuitiva claridad». La esencia

\footnotetext{
${ }^{31}$ Cfr., Ideas I, op. cit., 1913, 307; 1993², 333.

32 Al mencionar el contenido noemático del concepto que representa al "objeto" (digámoslo así) de la conciencia, Husserl mienta en concreto al objeto reconducido a la conciencia, a su puro sentido, a la síntesis predicativa pura que mienta al objeto. Por identificación con este nóema otros nóemas participan de su carácter de darse adecuadamente y de estar lleno y forman una unidad sintética noemática. Esta unidad posee, pues, certeza racional plena.

${ }^{33}$ San Martín, J., denomina a este proceso «variación imaginativa»; vid., La fenomenología de Husserl.., op. cit., 77.
} 
así aprehendida es «sujeto» (subyace invariablemente) de ulteriores determinaciones noemáticas. Ahora bien, la esencia que hemos alcanzado sin duda está dada originariamente, pero no de manera adecuada. ¿Cómo se soluciona el escollo toda vez que una intuición es susceptible de ser modificada por ulteriores modificaciones? Con la aprehensión de la Idea "cosa", aprehendemos otras ideas que son ingredientes de aquella Idea estáticamente: la idea de espacio, de tiempo y de materia. La Idea "cosa" y sus componentes ideales encierran la indeterminación de la esencia, contienen todas las infinitas variaciones y determinaciones noemáticas posibles.

Mas es la evidencia apodíctica ${ }^{34}$ la que garantiza la verificabilidad racional de la idea. Y ésta es susceptible de ser científicamente estudiada por cuanto se constituye en la conciencia como la unidad de algo de las series de diversos apareceres que es correlato de las relaciones noético-noemáticas.

\section{A MODO DE CONCLUSIÓN}

La fenomenología de la razón de Ideas no significa en modo alguno una huida hacia el mundo de la conciencia por mor de un supuesto desapego husserliano por el mundo real. Así lo reconoce el propio filósofo: el mundo del que se ocupa la fenomenología es el mundo natural y real, amén de todos los otros mundos que por medio de la epojé son re(con)ducidos y desconectados; se ocupa de ellos en tanto que «sentidos de un mundo» por medio de las implicaciones inherentes que relacionan la nóesis y el nóema ${ }^{35}$.

Hemos pretendido mostrar que, más allá de los prolijos análisis de las formaciones teóricas de la fenomenología, la razón se traduce, primero, como la esfera posibilitante de lo genuinamente humano, y, segundo, como el tópos determinante del mundo trascendental (Lebenswelt). Ambos, el sujeto trascendental y el mundo de la vida, conforman lo que nuestro filósofo denomina ciencia universal, y ello en la medida en que ambos no son sino el campo de investigación de la filosofía. «La ciencia trascendental -afirma Husserl- no es mundana» ${ }^{36}$, es decir, el carácter de la ciencia trascendental es trascendente, ya

${ }^{34}$ Cfr., Ideas, op. cit., 1913, 313; 19932, 359.

${ }^{35}$ Cfr., Ibíd., 1913, 302-303; 19932, 348.

${ }^{36}$ Lógica formal y trascendental. Ensayo de una crítica de la razón lógica (trad. de Luis Villoro), UNAM, México, 1962, 279. 
que abarca, por un lado, todo aquello que es propio del sujeto racional y, por otro, todo lo que ha sido constituido por tal sujeto. Que la ciencia universal no es mundana quiere decir que ésta se desarrolla en un plano superior al de los meros hechos. Mas también significa, y esto es extraordinariamente importante, que la ciencia universal se enmarca en el horizonte de sentido que la razón franquea.

No podemos perder de vista que el objetivo del proyecto fenomenológico de Husserl es recomponer un mundo que está enfrentado consigo mismo en virtud precisamente de un uso dogmático y no crítico de la razón. Ciertamente los hechos no se baten en duelo con las ideas, porque ambos pertenecen a un mismo sujeto, es decir, pertenecen subjetivamente al sujeto, y su posibilidad de existir proviene de la fuerza racional y de la consecuente capacidad constitutiva de tal sujeto. La fenomenología como teoría de la razón se va conformando como la solución eficaz y convincente al problema que planea el encasillamiento del ser humano y del mundo en la esfera de los meros hechos.

\section{BiBLIOGRAFÍA}

HUSSERL, E., Investigaciones lógicas I, Alianza Editorial, Madrid, 2009.

- Logische Untersuchungen. Esrter Theil. Prolegomena zur reinen Logik, [En línea]. Halle a. S., M. Niemeyer, 1900, http://www.freidok.uni-freiburg.de/volltexte/5920, 12.

- Ideas relativas a una fenomenología pura y a una filosofía fenomenológica. Libro I (trad. de José Gaos), FCE, México, $1993^{2}$.

- Ideen zu einer reinen Phänomenologie und phänomenologischen Philosophie. Erstes Buch: Allgemeine Einführung in die reine Phänomenologie [En línea]. Halle, M. Niemeyer, 1913, http://www.freidok.uni-freiburg.de/volltexte/5973, 278.

-Die Idee der Phänomenologie, Husserliana II, Herausgegeben und Eingeleitet von W. Biemel, den Haag, Martinus Nijhoff, 1950 (La idea de la fenomenología ((trad. de Miguel García-Baró), FCE, México, 2004).

- Lógica formal y trascendental. Ensayo de una crítica de la razón lógica (trad. de Luis Villoro), UNAM, México, 1962.

-Formale und transzendentale Logiek: Versusuch einer Kritik del logischen Vernunft [En línea]. Halle, M. Niemeyer, 1929, http://www.freidok.uni-freiburg.de/volltexte/6005/

LÓPEZ SÁNEZ, Ma C., Corrientes actuales de la filosofía I. En-clave fenomenológica, UNEDDykinson, Madrid, 2012.

SAN MARTÍN, J., La fenomenología como teoría de una racionalidad fuerte. Estructura y función de la fenomenología de Husserl y otros ensayos, UNED, Madrid, 1994.

- La fenomenología de Husserl como utopía de la razón. introducción a la fenomenología, Biblioteca Nueva, Madrid, 2008.

ZUBIRI, X., Escritos menores (1953-1983), Alianza Editorial - Fundación Zubiri, Madrid, 2007. 